\title{
PRODUCTS OF DERIVATIONS
}

\author{
by T. CREEDON
}

(Received 21st October 1996)

\begin{abstract}
We prove that if the product of two derivations on an algebra is a derivation, then the product maps the algebra into its nilradical. As a consequence we obtain a characterisation of when the product of two derivations on a semiprime algebra is a derivation. We also give a condition on two derivations on a Banach algebra which implies that their product has range contained in the Jacobson radical.
\end{abstract}

1991 Mathematics subject classification: 16W25, 47B47, 16N60, 46H99.

\section{Introduction}

An early result in the theory of derivations is the fundamental theorem of E. C. Posner [9, Theorem 1], proved in 1957, which asserts that if the product of two derivations on a prime ring of characteristic not two is a derivation, then one of the derivations must be zero. This is known as Posner's first theorem, and the proof is fairly short. The conclusion of this theorem does not hold if the characteristic of the ring is two. In the same paper Posner proved that if $d$ is a derivation on a prime ring $R$ such that the commutator $x d x-(d x) x$ is in the centre of $R$ for all $x \in R$, then either $d=0$ or $R$ is commutative. This is known as Posner's second theorem. The proof given by Posner is quite long. However, M. Mathieu [6] has shown that Posner's second theorem follows directly from Posner's first theorem if the characteristic of the ring is not equal to two. Posner's second theorem can be easily proven in the case where the ring is of characteristic two (see [1, p. 13]).

Considering the product of powers of derivations (on a prime ring) D. W. Jensen [4] has obtained extensions of Posner's first theorem. In this paper we extend Posner's first theorem to the case of semiprime algebras. We prove that if the product of two derivations on an algebra $A$ is a derivation, then the product maps the algebra into its nilradical $\operatorname{nil}(A)$ (the intersection of all prime ideals of $A$ ). It follows that if the product of two derivations on a semiprime algebra is a derivation, then the product is zero. We also give a condition which implies that the product of two derivations maps the algebra into its Jacobson radical (Proposition 9).

I would like to thank my supervisor Prof. G. J. Murphy for his helpful comments. 


\section{Results}

We will use the following result.

Lemma 1. $[3,3.3 .2]$ If $d$ is a derivation on an algebra $A$ and $P$ is a minimal prime ideal of $A$, then $d(P) \subseteq P$.

Any derivation is a Jordan derivation. J. M. Cusack [2, Corollary 5] has shown that any Jordan derivation on a semiprime algebra is a derivation, but he also gives examples of Jordan derivations which are not derivations.

Theorem 2. If $d$ and $\delta$ are derivations on an algebra $A$ such that the product $d \delta$ is $a$ Jordan derivation, then $d \delta(A) \subseteq \operatorname{nil}(A) \subseteq \operatorname{rad}(A)$.

Proof. Every prime ideal contains a minimal prime ideal [8]. Hence, nil $(A)$ is equal to the intersection of the minimal prime ideals of $A$. Let $P$ be a minimal prime ideal of $A$. By Lemma $1, d(P) \subseteq P$ and $\delta(P) \subseteq P$. Hence, we can consider the induced derivations $d_{P}$ and $\delta_{P}$ on the prime algebra $A / P$, defined by $d_{P}(a+P)=d a+P$ and $\delta_{P}(a+P)=\delta a+P$, for all $a \in A$. Since $d \delta$ is a Jordan derivation, by [2, Theorem 4], $d \delta(a b)-a d \delta b-(d \delta a) b \in \operatorname{nil}(A) \subseteq P$. Hence, $d_{P} \delta_{P}$ is a derivation on the prime algebra $A / P$. By Posner's first theorem, $d_{P}=0$ or $\delta_{P}=0$. Therefore $d \delta(A) \subseteq P$. Since this holds for each minimal prime ideal $P$, we see that $d \delta(A) \subseteq \operatorname{nil}(A)$.

Corollary 3. If $d$ and $\delta$ are derivations on a semiprime algebra $A$ such that the product $d \delta$ is a derivation, then $d \delta=0$.

Mathieu [5, Corollary 2] has proven that if the product of two derivations on a $C^{*}$-algebra is a derivation, then the product is zero. Corollary 3 is a generalisation (with a much easier proof) of his result. Using a similar method of proof to that of Theorem 2 we can prove the following:

Proposition 4. If $d$ is a derivation on an algebra $A$ such that $d^{2}$ is a Jordan derivation, then $d(A) \subseteq \operatorname{nil}(A) \subseteq \operatorname{rad}(A)$.

Corollary 5. If $d$ is a derivation on a semiprime algebra $A$ such that $d^{2}$ is also a derivation, then $d=0$.

Example 6. Let $A$ be the Banach subalgebra of $M_{2}(C)$ consisting of the upper triangular $2 \times 2$ matrices. Let $d$ be the derivation on $A$ defined by setting

$$
d\left(\begin{array}{ll}
x & y \\
0 & z
\end{array}\right)=\left[\left(\begin{array}{ll}
0 & 1 \\
0 & 1
\end{array}\right),\left(\begin{array}{ll}
x & y \\
0 & z
\end{array}\right)\right] .
$$

Now $d^{2}=0$ but clearly $d \neq 0$. This shows that Corollary 5 does not hold if we drop 
the assumption that the algebra is semiprime and Posner's first theorem does not hold if we drop the assumption that the ring is prime.

Example 7. Let $d$ be the derivation on $M_{2}(\mathbf{C})$ defined by

$$
d\left(\begin{array}{ll}
x & y \\
w & z
\end{array}\right)=\left[\left(\begin{array}{ll}
0 & 0 \\
1 & 0
\end{array}\right),\left(\begin{array}{ll}
x & y \\
w & z
\end{array}\right)\right] .
$$

Since $M_{2}(C)$ is primitive, it is also prime. We have $d^{3}=0$ and so $d^{3}$ is a derivation, but $d \neq 0$. Therefore Posner's first theorem does not hold for the product of three derivations.

Example 8. Let $d$ be a derivation on a $\mathrm{C}^{*}$-algebra $A$. Define a derivation $d^{*}$ by setting

$$
d^{*}(a)=\left(d\left(a^{*}\right)\right)^{*}, \quad(a \in A)
$$

Suppose the product $d^{*} d$ is a derivation. Let $x$ be a self-adjoint element of $A$. Now $(d x)(d x)^{*}+(d x)^{*}(d x)=0$. Setting $a=d x$, we have $a^{*} a=-a a^{*}$. Since the spectra $\sigma\left(a^{*} a\right)$ and $\sigma\left(a a^{*}\right)$ are both non-negative this implies that $a^{*} a=0$ and hence $a=0$. Therefore $d x=0$, for all self-adjoint elements $x \in A$. Since the self-adjoint elements of $A \operatorname{span} A$ we have $d=0$. So the product $d^{*} d$ is a derivation if and only if $d=0$.

We conclude with the following result.

Proposition 9. Suppose $\delta$ is a derivation (bounded or unbounded) on a Banach algebra $A$ and $d$ is a bounded derivation on $A$ such that $[d x, \delta y] \in \operatorname{rad}(A)$, for all $x, y \in A$. Then $d \delta(A) \subseteq \operatorname{rad}(A)$.

Proof. Let $P$ be any primitive ideal of $A$. Since $d$ is bounded, by [10, Theorem 2.2] we have $d(P) \subseteq P$ and we can consider the induced derivation $d_{P}$ on the primitive Banach algebra $A / P$. We have

$$
\left[\delta y+P, d_{P}(x+P)\right]=0, \text { for all } x, y \in A .
$$

Therefore, by Posner's first theorem, either $d_{P}(A / P)=0$ or $[\delta y+P, a+P]=0$, for all $y, a \in A$. So either $d(A) \subseteq P$ or $[\delta y, a] \in P$ for all $y, a \in A$. If $d(A) \subseteq P$, then in particular we have $d \delta(A) \subseteq P$. Suppose $[\delta y, a] \in P$, for all $y, a \in A$. Since $d$ is bounded, $d([\delta y, a]) \in P$, for all $y, a \in A$. That is, $d([\delta y, a])=[d \delta y, a]+[\delta y, d a] \in P$. Therefore, $[d \delta y, a] \in P$, for all $y, a \in A$, since by hypothesis $[\delta y, d a] \in P$. Therefore, $d_{P}(\delta y+P) \in Z(A / P) \cong \mathbf{C}$. Hence, $d_{P}^{2}(\delta y+P)=0$, and [7, Theorem 2.1] implies that $d_{P}(\delta y+P)$ is quasinilpotent. Since $d_{P}(\delta y+P)$ is both quasinilpotent and a scalar, it must equal zero. Therefore $d_{P}(\delta y+P)=0$, for all $y \in A$. Hence, $d \delta(A) \subseteq P$.

So in either case we have $d \delta(A) \subseteq P$. Since this holds for every primitive ideal $P$, the result follows. 


\section{REFERENCES}

1. M. Ahmad, On a theorem of Posner, Proc. Amer. Math. Soc. 66 (1977), 13-16.

2. J. M. Cusack, Jordan derivations on rings, Proc. Amer. Math. Soc. 53 (2) (1975), 321324.

3. J. Dixmier, Algèbres envellopantes (Cahier Sci. 27, Gauthier-Villars, Paris, 1974).

4. D. W. Jensen, Nilpotency of derivations in prime rings, Proc. Amer. Math. Soc. 123 (9) (1995), 2633-2636.

5. M. Mathieu, Properties of the product of two derivations of a $\mathrm{C}^{*}$-algebra, Canad. Math. Bull. 32 (4) (1989), 490-497.

6. M. Mathieu, Posner's second theorem deduced from the first, Proc. Amer. Math. Soc. 114 (1992), 601-602.

7. M. Mathieu and G. J. Murphy, Derivations mapping into the radical, Arch. Math. (Basel) 57 (1991), 485-487.

8. N. H. McCoy, Prime ideals in general rings, Amer. J. Math. 71 (1949), 823-833.

9. E. C. Posner, Derivations in prime rings, Proc. Amer. Math. Soc. 8 (1957), 1093-1100.

10. A. M. Sinclatr, Continuous derivations on Banach algebras, Proc. Amer. Math. Soc. 20 (1969), 166-170.

Department of Mathematics

UNIVERSiTy COLlege

CORK, IRELAND 